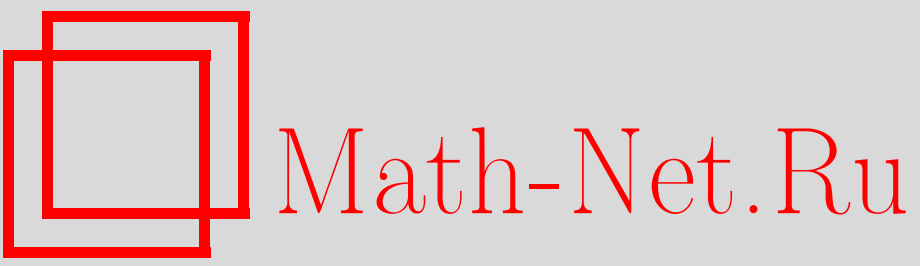

Ю. Кнежевич-Милянович, Оценки минимального собственного значения самосопряженных дифференциальных операторов высокого порядка, УМН, 2000, том 55, выпуск 2, 139-140

DOI: https://doi.org/10.4213/rm277

Использование Общероссийского математического портала Math-Net.Ru подразумевает, что вы прочитали и согласны с пользовательским соглашением

http://www. mathnet.ru/rus/agreement

Параметры загрузки :

IP: 35.174 .16 .151

26 апреля 2023 г., 16:08:05 


\section{ОЦЕНКИ МИНИМАЛЬНОГО СОБСТВЕННОГО ЗНАЧЕНИЯ САМОСОПРЯЖКНННХ ДИФФЕРЕНЦИАЛЬНЫХ ОПЕРАТОРОВ ВЫСОКОГО ПОРЯДКА}

Ю. КнЕЖЕВИЧ-Милянович

Рассматривается краевая задача:

$$
\begin{gathered}
\frac{d^{n}}{d x^{n}}\left(p(x) \frac{d^{n} y}{d x^{n}}\right)=(-1)^{n} \lambda y, \quad a<x<b, \\
y^{(i)}(a)=0, \quad y^{(i)}(b)=0, \quad 1 \leqslant i \leqslant n-1,
\end{gathered}
$$

где $p(x)$ - измеримая функция, которая на $[a, b]$ ограничена и строго положительна. Как известно, спектр задачи (1) положительный и дискретный:

$$
0<\lambda_{1}(p(x))<\lambda_{2} \leqslant \lambda_{3} \leqslant \cdots .
$$

Зафикисируем $\alpha \neq 0$ и предположим, что

$$
\int_{a}^{b} p^{\alpha}(x) d x=1
$$

Будет изучаться вопрос об оценках

$$
c_{\alpha}<\lambda_{1} \leqslant C_{\alpha}
$$

в классе задач с коэффициентом $p(x)$, удовлетворяющим (2). При $n=1$ такая задача полностью исследована в работах [1], [2]. Для операторов высшего порядка вида

$$
\frac{d^{2 n} y}{d x^{2 n}}=(-1)^{n} \lambda p(x) y
$$

такой вопрос рассматривался также в [1], [3]. Методы предшествуюших работ не распространяются без существенных изменений на случай задачи (1) с $n>1$.

Основной результат настоящей работы:

Теорема 1. Если $\alpha>-\frac{1}{2 n}$, то существует константа $C_{\alpha}<\infty$ такал, что $\lambda_{1}<C_{\alpha}$. Если $\alpha \leqslant-1$, то существует $c_{\alpha}>0$ такая, что $\lambda_{1}>c_{\alpha}$.

Можно, кроме того, показать, что если $\alpha \leqslant-\frac{1}{2 n}$, то $\lambda_{1}$ может принимать сколь угодно малые положительные значения, а если $\alpha>-1$, то $\lambda_{1}$ может принимать сколь угодно большие положительные значения.

ДокАЗАТЕЛЬСтво тЕОРЕМЫ 1. Доказательство основано на применении вариационного принципа:

$$
\lambda_{1} \leqslant J(y)=\left(\int_{a}^{b} p(x) y^{(n)^{2}} d x\right)\left(\int_{a}^{b} y^{2} d x\right)^{-1}
$$

где $y \in \stackrel{\circ}{W} \underset{2}{n}([a, b]), y \not \equiv 0$.

Пусть $-\frac{1}{2 n}<\alpha<1$. Будем искать $y_{1}(x) \in \stackrel{\circ}{W_{2}^{n}}([a, b])$, так что

$$
y_{1}^{(n)^{2}}=p(x)^{\alpha-1} \text {. }
$$

Пусть $a=\xi_{0}<\xi_{1}<\cdots<\xi_{n}<b=\xi_{n+1}$. Ограничимся рассмотрением случая $a=0$, $b=1$. Функцию $y_{1}$ определим как решение уравнения $y_{1}^{(n)}=\Theta(x) p(x)^{\frac{\alpha-1}{2}}$, где $\Theta(x)$ - ступенчатая функция, принимающая на каждом интервале $\left(\xi_{i}, \xi_{i+1}\right)$ значения или 1 или -1 и $y_{1}(0)=$ 
$y_{1}^{\prime}(0)=\cdots=y_{1}^{(n-1)}(0)=1$. Набору $\left(\xi_{0}, \xi_{1}, \ldots, \xi_{n}\right)$ и функции $\Theta(x)$ поставим в соответствие точку $\pm\left(\xi_{i+1},-\xi_{i}\right)$, где знак “+" берется в случае, когда $\Theta(x)=1$ на $\left(\xi_{i+1}, \xi_{i}\right)$, и “-", если $\Theta(x)=-1$ на этом интервале. Заметим, что образ набора $\left(\xi_{0}, \ldots, \xi_{n}\right)$ и функции $\Theta(x)$ заполнят полиэдр $\left|t_{0}\right|+\left|t_{1}\right|+\cdots+\left|t_{n}\right|=1$ в $\mathbb{R}^{n+1}$ и такое соответствие взаимно однозначно. Далее, каждой точке $\left(t_{0}, \ldots, t_{n}\right)$ поставим в соответствие точку $\left(y_{1}(1), y_{1}^{\prime}(1), \ldots, y_{1}^{(n-1)}(1)\right)$ гиперплоскости $\mathbb{R}^{n}$. Получено нечетное отображение полиэдра на гиперплоскость. Из теоремы Борсука [4] следует, что некоторая точка полиэдра отображается в начало координат. Такой точке соответствует решение $y_{1}(x)$ уравнения $(4)$ из $\stackrel{\circ}{W} \underset{2}{n}([a, b])$. Эта функция будет рассматриваться как пробная функция в [3]. Далее используем лемму, доказательство которой достаточно элементарно и будет здесь опущено.

Лемма 1. Если $y(x) \in C^{n}\left(\left[a_{1}, a_{2}\right]\right), y^{(n)}$ не меняет знака, $s<\frac{2}{1+2 n}$, то

$$
\left(\int_{a_{1}}^{a_{2}}\left|y^{(n)}(x)\right|^{s} d x\right)^{1 / s} \leqslant C\left|a_{2}-a_{1}\right|^{\frac{2-s-2 k s}{2 s}}\left(\int_{a_{1}}^{a_{2}} y^{2} d x\right)^{1 / 2} .
$$

Предположим сейчас, что $0<\alpha<1$. Применим лемму 1 к построенной выше функции $y_{1}(x)$ с $k=n, s=\frac{2 \alpha}{\alpha-1}$ на самом большом из интервалов $\left(\xi_{1}, \xi_{i+1}\right), i=0, \ldots, n-1$. В результате
получим, что

$$
\int_{a}^{b}\left|y_{1}\right|^{2} d x \geqslant C_{1}(\alpha)
$$

Отсюда и из (4) при $y=y_{1}$ следует, что $\lambda_{1} \leqslant C_{\alpha}$. Если же $-\frac{1}{2 n}<\alpha<0$, то лемму 1 следует применить на самом маленьком интервале среди $\left(\xi_{i}, \xi_{i+1}\right), i=1, \ldots, n$.

Утверждение леммы 1 при $\alpha \geqslant 1$ следует из (4), если взять в качестве $y$ любую функцию из $\stackrel{\circ}{C}^{\infty}(a, b)$, не равную нулю тождественно, и применить неравенство Гёльдера. Чтобы получить утверждение для $\alpha \leqslant-1$, достаточно рассмотреть случай $\alpha=-1$. В таком случае:

$$
\left(\int_{a}^{b} y^{2} d x\right)^{1 / 2} \leqslant c \int_{a}^{b}\left|y^{(n)}\right| d x \leqslant c \int_{a}^{b} p(x) y^{(n)^{2}} d x
$$

следовательно, $J(y) \geqslant C_{\alpha}$ и $\lambda_{1} \geqslant C_{\alpha}$ в силу вариационного принципа.

Переходим к рассмотрению случая $\alpha \geqslant 1$. В этом случае $\lambda_{1}$ может принимать сколь угодно малые положительные значения. Чтобы убедиться в этом, положим $a=-1, b=1, p_{0}(x)=|x|$. Ясно, что $J\left(y\left(\varepsilon^{-1} x\right)\right) \rightarrow 0$ при $\varepsilon \rightarrow 0$, какой бы ни была $y(x) \in \stackrel{\circ}{C^{\infty}}(-1,1)$. В таком случае, если $p=p_{0}(x)+\delta^{2}$, то $\lambda_{i}(p(x))$ будет стремиться к нулю при $\delta \rightarrow 0$, оставаясь положительным. Осталось показать, что если $\alpha \leqslant-\frac{1}{2 n}$, то $\lambda_{1}$ может принимать сколь угодно большие значения. Это делается при произвольном $n$ так же, как и при $n=1$ в работе [2]. Заметим еще, что в классе краевых задач с $p(x)$, удовлетворяющих (2), и $\alpha>-\frac{1}{2 n}$ существует $P(x)$ такая, что $\lambda_{1} \leqslant \lambda_{1}(P(x))$. Такая $P(x)$ непрерывна и положительна на $(a, b)$ и также удовлетворяет $(2)$.

\section{СПИСОК ЛИТЕРАТУРЫ}

[1] Егоров Ю. В., Кондратьев В. А. Об оценках первого собственного значения в некоторых задачах Штурма-Лиувилля // УМН. 1996. Т. 51. № 3. С. 73-144. [2] Egorov Yu. V., Karaa S. Optimization de la premiere valeur propre de l'operateur de SturmLiouville // C. R. Acad. Sci. Paris Sér. I. 1994. V. 319. P. 793-798. [3] Егоров Ю. В., Кондратьев В. А. Об оценках первого собственного значения задачи Штурма-Лиувилля // УМН. 1984. Т. 39. № 2. С. 151-152. [4] Тихомиров В. М. Теория приближений. 